\title{
Generalized Yang-Mills Theory and Gravity
}

\author{
Pei-Ming $\mathrm{Ho}^{1}$, \\ Department of Physics and Center for Theoretical Sciences \\ Center for Advanced Study in Theoretical Sciences \\ National Taiwan University, Taipei 106, Taiwan, R.O.C.
}

\begin{abstract}
We propose a generalization of Yang-Mills theory for which the symmetry algebra does not have to be factorized as mutually commuting algebras of a finite-dimensional Lie algebra and the algebra of functions on base space. The algebra of diffeomorphism can be constructed as an example, and a class of gravity theories can be interpreted as generalized Yang-Mills theories. These theories in general include a graviton, a dilaton and a rank-2 antisymmetric field, although Einstein gravity is also included as a special case. We present calculations suggesting that the connection in scattering amplitudes between Yang-Mills theory and gravity via BCJ duality can be made more manifest in this formulation.
\end{abstract}

\footnotetext{
${ }^{1}$ e-mail address: pmho@phys.ntu.edu.tw
} 


\section{Introduction}

The textbook definition of Yang-Mills (YM) theory is usually based on the choice of a finite dimensional Lie group, and gauge transformations are specified by Lie-group valued functions. However, it is well known that when the base space is noncommutative, the algebra of gauge transformations is a mixture of the finite-dimensional Lie algebra and the algebra of functions on the noncommutative space. As a result, $S U(N)$ gauge symmetry cannot be straightforwardly defined on noncommutative space.

In this paper, we consider a minor generalization of the notion of gauge symmetry. We will not only allow the generators of gauge transformations to behave like pseudo-differential operators (as functions on noncommutative space do), but we will also allow them to be not factorized into the part of a finite-dimensional Lie algebra and that of functions on the base space. That is, the gauge symmetry algebra does not have to be defined as the composition of a finite-dimensional Lie algebra and an associative algebra of functions on the base space. With this generalization, it may no longer be possible to view a gauge symmetry as what you get from "gauging" a global symmetry through introducing space-time dependence.

A possibility of this generalization was already suggested [1] for even-dimensional spherical brane configurations in the matrix theory. For example, for the fuzzy- $S^{4}$ configuration of $n$ D4-branes, the algebra of functions on the 4-dimensional base space is non-associative, but there is an associative algebra for gauge transformations. For large $n$, the gauge symmetry algebra is approximately that of a $U(n)$-bundle (or equivalently a fuzzy- $S^{2}$ bundle) over $S^{4}[1]$.

Another example is the low energy effective theory of a D3-brane in large R-R 2-form field background [2]. This theory is S-dual to the noncommutative gauge theory for a D3-brane in large NS-NS B-field background. The gauge symmetry to all orders in the dual theory is not given by the noncommutative gauge symmetry, but is characterized by a bracket $\{\cdot, \cdot\}_{* *}$ which defines a non-associative algebra on the base space [2]. (The gauge symmetry algebra is of course associative.)

In this paper, we will show that the gauge symmetry of space-time diffeomorphism is also an example of the generalized gauge symmetry. Accordingly, a class of gravity theories can be interpreted as YM theories. Generically, these theories include a graviton, a dilaton and an anti-symmetric tensor. We will point out that the connection between Yang-Mills theory and gravity (through the color-kinematics duality) is manifest at tree level in 3-point amplitudes.

Attempts to interpret gravity as a gauge theory have a long history since the works of Utiyama [3], Kibble [4] and Sciama [5]. It is well known that General Relativity (GR) can be rewritten as the Chern-Simons theory in 3 dimensions [6], and a YM-like theory in 4 dimensions [7, 8], as well as higher dimensions [9]. The vielbein and the connection are defined as components of a gauge potential, and the gauge symmetry is $S O(d, 2)$, instead of the space-time diffeomorphism. These formulations are based on gauge symmetries in 
the traditional sense. Our formulation of gravity as a YM theory is different from these formulations.

While GR can be formulated as a YM theory, YM theories can also be realized as the low energy effective theories of gravity theories in higher dimensions via suitable compactification. Similar to this scenario of Kaluza-Klein reduction, internal symmetries and external symmetries are treated on equal footing in the generalized YM theories, as we will not distinguish the base space dependence from the internal space dependence in the gauge symmetry algebra.

Our formulation of gravity is also reminiscent of teleparallel gravity [10], which can be interpreted as a gauge theory of the (Abelian) translation group, with the vielbeins playing the role of the gauge potential. In another formulation of gravity [11] in which the vielbeins are identified with the gauge potential, a deformation of the gauge symmetry is considered to achieve the nonlinearity in gravity. In our formulation, on the other hand, the gauge potential is not the vielbein, but the inverse of the vielbein.

The plan of this paper is as follows. In Sec.2, we will see how the algebra of diffeomorphism appears as an example of the generalized gauge symmetry. In Sec.3, the gauge potential for the gauge symmetry of diffeomorphism is essentially the inverse of the vielbein, and the field strength is the torsion of the Weitzenböck connection. We show that the corresponding YM theories with quadratic Lagrangians define a class of gravity theories in Sec.4. It will be pointed out in Sec.5 that this new formulation of gravity may have significant advantages in its use to compute scattering amplitudes, with relations reminiscent of the double-copy procedure [13] to derive scattering amplitudes in gravity from YM theories. In Sec.6, we comment on extensions of the generalized notion of gauge symmetry to higher form gauge theories.

\section{Gauge Symmetry Algebra}

In a naive textbook introduction to non-Abelian gauge symmetry, the gauge transformation parameter $\Lambda(x)=\sum_{a} \Lambda^{a}(x) T_{a}$ is a sum of products of space-time functions and Lie algebra generators. The Lie algebra of local gauge transformations is spanned by a set of basis elements, say,

$$
T_{a}(p) \equiv e^{i p \cdot x} T_{a}
$$

in the Fourier basis. In this basis, a gauge transformation parameter can be expressed as

$$
\Lambda(x)=\sum_{a, p} \tilde{\Lambda}^{a}(p) T_{a}(p)
$$

where the sum over $p$ is understood to be the integral $\int d^{D} p$ for $D$-dimensional space-time. Similarly, the gauge potential can be written as

$$
A_{\mu}(x)=\sum_{a, p} \tilde{A}_{\mu}^{a}(p) T_{a}(p)
$$


Normally, for a given finite-dimensional Lie algebra with structure constants $f_{a b}{ }^{c}$, the algebra of gauge transformations has the commutator

$$
\left[T_{a}(p), T_{b}\left(p^{\prime}\right)\right]=\sum_{c} f_{a b}{ }^{c} T_{c}\left(p+p^{\prime}\right)
$$

where the structure constants $f_{a b}{ }^{c}$ only involve color indices $a, b, c$. For these cases, the inclusion of functional dependence on the space-time in the generators $T_{a}(p)$ is trivial, and thus often omitted in discussions.

However, for noncommutative gauge symmetries, the structure constants depend not only on the color indices $a, b, c$, but also on the kinematic parameters $p, p^{\prime}$. For a noncommutative space defined by

$$
\left[x^{\mu}, x^{\nu}\right]=i \theta^{\mu \nu}
$$

the Lie algebra $U(N)$ gauge symmetry is

$$
\left[T_{a}(p), T_{b}\left(p^{\prime}\right)\right]=\sum_{p^{\prime \prime}} \mathbf{f}_{a b}^{c}\left(p, p^{\prime}, p^{\prime \prime}\right) T_{c}\left(p^{\prime \prime}\right)
$$

where the structure constants are ${ }^{1}$

$$
\mathbf{f}_{a b}^{c}\left(p, p^{\prime}, p^{\prime \prime}\right)=\left[f_{a b}^{c}\left(e^{i p \theta p^{\prime}}-e^{i p^{\prime} \theta p}\right)+d_{a b}^{c}\left(e^{i p \theta p^{\prime}}+e^{i p^{\prime} \theta p}\right)\right] \delta^{(D)}\left(p+p^{\prime}-p^{\prime \prime}\right),
$$

and they involve kinematic parameters $p, p^{\prime}$ and $p^{\prime \prime}$. Here $f_{a b}{ }^{c}$ is the structure constant of $U(N)$ and $d_{a b}^{c}$ is defined by

$$
\left\{T_{a}, T_{b}\right\}=d_{a b}{ }^{c} T_{c}
$$

for $T_{a}$ 's in the fundamental representation. In this gauge symmetry algebra, the $U(N)$ Lie algebra and the algebra of functions on the base space are mixed. (This is the obstacle to define noncommutative $S U(N)$ gauge symmetry.) The gauge algebra is non-Abelian even for the Abelian group $U(1)$.

To describe the noncommutative $U(N)$ gauge algebra properly, it is a necessity to use the generators (1) including functional dependence on the base space. Nevertheless, the noncommutative $U(N)$ gauge symmetry still assumes that the generators can be factorized (1), and that $e^{i p \cdot x}$ always commutes with $T_{a}$. These are unnecessary assumptions for most algebraic calculations in the gauge theory. After all, in field theories, only the coefficients $\tilde{A}_{\mu}^{a}(p)$ are operators (observables), while the space-time and Lie algebra dependence are to be integrated out (summed over) in the action.

It is thus natural to slightly extend the formulation of gauge symmetry (and YM theory) to allow the Lie algebra to be directly defined in terms of the generators $T_{a}(p)$, without even assuming its factorization into a Lie algebra factor $T_{a}$ and a function $e^{p \cdot x}$. The integration over space-time and trace of the internal space in the action can be replaced by the Killing form of the Lie algebra of $T_{a}(p)$. The distinction between internal space and external space is reduced in this description.

\footnotetext{
${ }^{1}$ Here $p \theta p^{\prime}$ stands for $p_{\mu} \theta^{\mu \nu} p_{\nu}^{\prime}$.
} 
In short, we propose to study gauge symmetries without assuming its factorization into two associative algebras (an algebra for the functions on the base space and a finite dimensional Lie algebra). Even when it is possible to factorize the generators formally as (1), we will not assume that the space-time functions to commute with the algebraic elements $T_{a}$. (In general, we do not have to use the Fourier basis, and the argument $p$ of $T_{a}(p)$ can represent labels of any complete basis of functions on the base space.) One of the goals of this paper is to show that this generalization is beneficial, for bringing in new insights into gravity theories.

Algebraically, this generalization is very natural. A corresponding geometric notion is however absent at this moment. (It is not clear what twisted bundles would mean.) The notion of bundles on noncommutative space is replaced by projective modules [15], which should be further generalized for our purpose. We postpone the problem of finding a suitable geometric notion for the generalized gauge symmetry to the future.

\subsection{A Generalized Gauge Symmetry Algebra}

For the generalized gauge symmetry, we consider a Lie algebra defined as

$$
\left[T_{a}(\alpha), T_{b}(\beta)\right]=\sum_{\gamma} f_{a b}^{c}(\alpha, \beta, \gamma) T_{c}(\gamma)
$$

which may not be decomposed as a product of the algebra of functions on the space-time and a finite-dimensional Lie algebra. Here $\alpha, \beta, \gamma$ are labels for a complete basis of functions on the base space. For a theory with translational symmetry, it would be natural to use the Fourier basis, and we have

$$
\left[T_{a}(p), T_{b}\left(p^{\prime}\right)\right]=f_{a b}^{c}\left(p, p^{\prime}\right) T_{c}\left(p+p^{\prime}\right)
$$

where the structure constant is actually $f_{a b}{ }^{c}\left(p, p^{\prime}\right) \delta^{(D)}\left(p+p^{\prime}-p^{\prime \prime}\right)$ with the Dirac delta function cancelled by the integration over $p^{\prime \prime}$.

The Jacobi identity of this Lie algebra is

$$
f_{a b}^{e}\left(p, p^{\prime}\right) f_{e c}{ }^{d}\left(p+p^{\prime}, p^{\prime \prime}\right)+f_{b c}^{e}\left(p^{\prime}, p^{\prime \prime}\right) f_{e a}{ }^{d}\left(p^{\prime}+p^{\prime \prime}, p\right)+f_{c a}^{e}\left(p^{\prime \prime}, p\right) f_{e b}{ }^{d}\left(p^{\prime \prime}+p, p^{\prime}\right)=0 .
$$

Every solution to this equation for $f$ defines a gauge symmetry. It will be interesting to find solutions with non-trivial dependence on momenta, as the case of the noncommutative gauge symmetry.

As an example, let us now construct a Lie algebra for generators of the form

$$
\left\{T(\tilde{\epsilon}, p) \equiv \tilde{\epsilon}^{(\mu)} T_{(\mu)}(p)\right\}
$$

where the basis elements $T_{(\mu)}(p)$ has a space-time index $\mu$ as its internal space index. To construct a concrete example, we will assume that the structure constants are linear in momenta $p, p^{\prime}$, and that it is compatible with Poincare symmetry. 
We put the index $\mu$ in a parenthesis to remind ourselves that it plays the role of the index of an internal space. The reason why we choose this ansatz for a Lie algebra is that the Lie algebra of space-time diffeomorphisms is of this form. We wish to explore the possibility of rewriting a gravity theory as a generalized YM theory.

The translation symmetry implies that the commutators are of the form

$$
\left[T\left(\tilde{\epsilon}_{1}, p_{1}\right), T\left(\tilde{\epsilon}_{2}, p_{2}\right)\right]=\sum_{\tilde{\epsilon}_{3}} f\left(\tilde{\epsilon}_{1}, p_{1}, \tilde{\epsilon}_{2}, p_{2}, \tilde{\epsilon}_{3}\right) T\left(\tilde{\epsilon}_{3}, p_{1}+p_{2}\right)
$$

and the Lorentz symmetry implies that the structure constants are Lorentz-invariant functions of the vectors $\tilde{\epsilon}_{1}, \tilde{\epsilon}_{2}, \tilde{\epsilon}_{3}, p_{1}, p_{2}$. (The summation over $\tilde{\epsilon}_{3}$ is a summation over an orthonormal basis of vectors.) The most general ansatz consistent with all assumptions is thus

$f\left(\tilde{\epsilon}_{1}, p_{1}, \tilde{\epsilon}_{2}, p_{2}, \tilde{\epsilon}_{3}\right)=\left(\tilde{\epsilon}_{1} \cdot \tilde{\epsilon}_{3}\right)\left[\tilde{\epsilon}_{2} \cdot\left(\alpha p_{1}+\gamma p_{2}\right)\right]-\left(\tilde{\epsilon}_{2} \cdot \tilde{\epsilon}_{3}\right)\left[\tilde{\epsilon}_{1} \cdot\left(\alpha p_{2}+\gamma p_{1}\right)\right]+\beta\left(\tilde{\epsilon}_{1} \cdot \tilde{\epsilon}_{2}\right)\left[\tilde{\epsilon}_{3} \cdot\left(p_{1}-p_{2}\right)\right]$,

where $\alpha, \beta, \gamma$ are constant parameters.

It follows from the ansatz that

$$
\begin{aligned}
& {[[T(1), T(2)], T(3)]+[[T(2), T(3)], T(1)]+[[T(3), T(1)], T(2)]=\sum_{\tilde{\epsilon}_{4}}\{} \\
& \left(\tilde{\epsilon}_{1} \cdot \tilde{\epsilon}_{4}\right)\left[-\alpha \beta\left(\tilde{\epsilon}_{2} \cdot \tilde{\epsilon}_{3}\right)\left(p_{1} \cdot\left(p_{2}-p_{3}\right)\right)+\gamma\left[\left(\tilde{\epsilon}_{2} \cdot p_{2}\right)\left(\tilde{\epsilon}_{3} \cdot\left(\alpha p_{1}+\gamma p_{2}\right)-\left(\tilde{\epsilon}_{3} \cdot p_{3}\right)\left(\tilde{\epsilon}_{2} \cdot\left(\alpha p_{1}+\gamma p_{3}\right)\right]\right]\right.\right. \\
& +\beta\left(\tilde{\epsilon}_{1} \cdot \tilde{\epsilon}_{2}\right)\left[\left(\tilde{\epsilon}_{3} \cdot\left(p_{1}-p_{2}\right)\right)\left(\tilde{\epsilon}_{4} \cdot\left[(\alpha-\beta)\left(p_{1}+p_{2}\right)-\beta\left(p_{1}-p_{2}\right)\right]\right)-\gamma\left(\tilde{\epsilon}_{3} \cdot p_{3}\right)\left(\tilde{\epsilon}_{4} \cdot\left(p_{1}-p_{2}\right)\right)\right] \\
& + \text { cyclic permutations of }(1,2,3)\},
\end{aligned}
$$

where we have used $T(1)$ to represent $T\left(\tilde{\epsilon}_{1}, p_{1}\right), T(2)$ to represent $T\left(\tilde{\epsilon}_{2}, p_{2}\right)$, etc. In order to satisfy the Jacobi identity, we have to set $\beta=\gamma=0$. The most general solution is thus equivalent to

$$
[T(1), T(2)]=\sum_{\tilde{\epsilon}_{3}} i\left[\left(\tilde{\epsilon}_{2} \cdot \tilde{\epsilon}_{3}\right)\left(\tilde{\epsilon}_{1} \cdot p_{2}\right)-\left(\tilde{\epsilon}_{1} \cdot \tilde{\epsilon}_{3}\right)\left(\tilde{\epsilon}_{2} \cdot p_{1}\right)\right] T(3)
$$

by scaling $\alpha$ to $-i$. More explicitly, it is

$$
\left[T_{(\mu)}\left(p_{1}\right), T_{(\nu)}\left(p_{2}\right)\right]=\sum_{\lambda} i\left[\eta_{\nu \lambda} p_{2 \mu}-\eta_{\mu \lambda} p_{1 \nu}\right] T_{(\lambda)}\left(p_{1}+p_{2}\right)
$$

Incidentally, it is consistent to allow $\alpha$ to depend on the momenta. For example, Jacobi identity is satisfied for

$$
\alpha\left(p_{1}, p_{2}\right)=c e^{\lambda p_{1} \cdot p_{2}}
$$

for arbitrary constant parameters $c$ and $\lambda$. It is equivalent to the scaling of the generators by $T(\epsilon, p) \rightarrow T^{\prime}(\epsilon, p) \equiv c e^{\lambda p^{2} / 2} T(\epsilon, p)$. 


\subsection{Representations}

A representation of the algebra constructed above is given by

$$
T(\tilde{\epsilon}, p) \tilde{\phi}\left(p^{\prime}\right)=i\left(\tilde{\epsilon} \cdot p^{\prime}\right) \tilde{\phi}\left(p^{\prime}+p\right)
$$

on a linear space with the basis $\{\tilde{\phi}(p)\}$. This expression allows us to interpret $\tilde{\phi}(p)$ as the Fourier modes of a scalar field $\phi(x)$ and $T(\tilde{\epsilon}, p)$ as the generator of a coordinate transformation with

$$
\delta x^{\mu}=\tilde{\epsilon}^{(\mu)} e^{-i p \cdot x} .
$$

That is,

$$
T(\tilde{\epsilon}, p)=e^{i p_{\nu} x^{\nu}} \tilde{\epsilon}^{(\mu)} \partial_{\mu} .
$$

Indices are contracted according to Einstein's summation convention regardless of whether they are in parentheses or not. In this representation, it is clear that this algebra is that of space-time diffeomorphism. The gauge symmetry of GR arises as the most general gauge symmetry with Lie algebra of the form (13), assuming that the structure constants are linear in momentum and that they respect Poincare symmetry.

A generic element of the Lie algebra is a superposition

$$
\int d^{D} p \tilde{\epsilon}^{(\mu)}(p) T_{(\mu)}(p)
$$

in $D$ dimensions, which can be written as

$$
T_{\epsilon} \equiv \epsilon^{(\mu)}(x) \partial_{\mu}
$$

where $\epsilon^{(\mu)}(x)$ is the inverse Fourier transform of $\tilde{\epsilon}^{(m)}(p)$.

In view of this representation (23), it is tempting to interpret the algebra constructed above as merely the result of taking $T_{a}$ 's to be derivatives $\partial_{a}$ 's in (1) for a traditional gauge symmetry. But if we were really dealing with a traditional gauge symmetry, we would have obtained an Abelian gauge symmetry because $\left[T_{a}, T_{b}\right]=\left[\partial_{a}, \partial_{b}\right]=0$. The need to generalize the notion of gauge symmetry here is due to the fact that $T_{a}=\partial_{a}$ does not commute with space-time functions. Incidentally, the traditional interpretation of the torsion in teleparallel gravity is indeed the field strength of an Abelian gauge theory [14]. (See eq.(55) below.)

Matter fields in the gauge theory are classified as representations of the gauge symmetry. Since the gauge symmetry under consideration is the diffeomorphism, we know all about other representations of different spins. 


\section{Gauge Field of Diffeomorphism}

\subsection{Gauge Potential vs Vielbein}

We can define a gauge potential for the gauge symmetry algebra (16). In the representation (23), the gauge potential

$$
A_{\mu}(x)=\sum_{\nu, p} \tilde{A}_{\mu}^{(\nu)}(p) T_{(\nu)}(p)=A_{\mu}^{(\nu)}(x) \partial_{\nu}
$$

should transform like

$$
\delta A_{\mu}(x)=\left[D_{\mu}, \Lambda(x)\right]
$$

where

$$
D_{\mu} \equiv \partial_{\mu}+A_{\mu}(x)=\left(\delta_{\mu}^{\nu}+A_{\mu}^{(\nu)}\right) \partial_{\nu}
$$

is the covariant derivative and the gauge transformation parameter is

$$
\Lambda(x)=\Lambda^{(\mu)}(x) \partial_{\mu} .
$$

More explicitly, the gauge transformation (25) can be expressed as

$$
\delta A_{\mu}^{(\nu)}(x)=\partial_{\mu} \Lambda^{(\nu)}(x)-\Lambda^{(\lambda)}(x) \partial_{\lambda} A_{\mu}^{(\nu)}(x)+A_{\mu}^{(\lambda)}(x) \partial_{\lambda} \Lambda^{(\nu)}(x) .
$$

Let us recall that the vielbein $e_{\mu}{ }^{a}(x)$ in gravity is defined to transform under general coordinate transformations as

$$
\delta e_{\mu}{ }^{a}(x)=\delta x^{\nu} \partial_{\nu} e_{\mu}{ }^{a}+e_{\nu}{ }^{a} \partial_{\mu} \delta x^{\nu}
$$

The index $a$ on $e_{\mu}{ }^{a}$ labels a local orthonormal Lorentz frame. Under a rotation of the local Lorentz frame,

$$
e_{\mu}^{a}(x) \rightarrow e_{\mu}^{\prime a}(x)=\omega_{b}^{a}(x) e_{\mu}^{b}(x)
$$

where

$$
\omega_{a b}(x)=-\omega_{b a}(x)
$$

is the parameter for infinitesimal $S O(D-1,1)$-rotations.

The inverse $e_{a}{ }^{\mu}(x)$ of the vielbein is defined by

$$
e_{a}^{\mu}(x) e_{\mu}^{b}(x)=\delta_{a}^{b}, \quad e_{\mu}^{a}(x) e_{a}^{\nu}(x)=\delta_{\mu}^{\nu}
$$

The transformation of $e_{a}^{\mu}(x)$ is

$$
\delta e_{a}^{\mu}(x)=\delta x^{\nu} \partial_{\nu} e_{a}^{\mu}(x)-e_{a}{ }^{\nu} \partial_{\nu} \delta x^{\mu} .
$$

Let us now consider a flat background in which

$$
e_{a}^{\mu}(x)=\delta_{a}^{\mu}+C_{a}{ }^{\mu}(x)
$$


for a fluctuation denoted by $C_{a}{ }^{\mu}$. Here we have chosen a particular frame in which the flat background is given by $e_{a}{ }^{\mu}=\delta_{a}^{\mu}$. The local Lorentz transformation symmetry is not manifest (nonlinearly realized) in terms of the variable $C_{a}{ }^{\mu}$.

It follows from (33) that

$$
\delta C_{a}^{\mu}(x)=-\partial_{a} \delta x^{\mu}+\delta x^{\nu} \partial_{\nu} C_{a}^{\mu}(x)-C_{a}^{\nu} \partial_{\nu} \delta x^{\mu} .
$$

Comparing this expression with (28), we see that it is tempting to identify $A$ with $C$, and $\Lambda$ with $-\delta x$.

The transformations of $A$ and $C$ are matched by identifying upper (lower) indices with upper (lower) indices. That is, the Lorentz index $\mu$ on $A_{\mu}{ }^{(\nu)}$ is to be identified with the local Lorentz frame index $a$ on $C_{a}{ }^{\mu}$, while the internal space index $\nu$ on on $A_{\mu}{ }^{(\nu)}$ is to be identified with the space-time coordinate index $\mu$ on $C_{a}{ }^{\mu}$. This may seem peculiar at first sight but it is actually expected. The gauge algebra (16) is defined with the assumption of Poincare symmetry on the base space, so the Lorentz index $\mu$ on the potential $A_{\mu}{ }^{(\nu)}$ cannot be identified with the coordinate index $\mu$ on a curved manifold. On the other hand, the internal space index $\nu$ on the potential $A_{\mu}{ }^{(\nu)}$ is contracted with the index of a derivative $\partial_{\nu}$ of space-time coordinates, hence it is really an index of coordinates.

Note that the gauge potential $A$ is still defined as part of the covariant derivative $D=$ $d x^{\mu} D_{\mu}$, and in this sense it is still a 1-form. The one-form index $\mu$ of $A_{\mu}{ }^{(\nu)}$ is matched with the frame index, not the 1 -form index of the vielbein, only because the geometric interpretation of gravity is changed. The gauge symmetry of gravity is now interpreted as a non-Abelian symmetry on Minkowski space whose transformations involve kinematic vectors. On the other hand, the potential $A_{\mu}{ }^{(\nu)}$ is not a pure 1 -form as it has a vector-field index $(\nu)$.

In the following, we will adopt the conventional notation for vielbeins. Latin letters $a, b, c, \cdots$ are used for indices of local Lorentz frames, and Greek letters $\mu, \nu, \lambda, \cdots$ for indices of space-time coordinates. For instance, we will relabel the gauge potential as $A_{a}{ }^{(\mu)}$ (without raising or lowering indices), or simply as $A_{a}{ }^{\mu}$ without the parenthesis.

Despite the fact that $A$ and $C$ transform in exactly the same way under general coordinate transformations, it is not clear yet whether $A$ can be fully identified with $C$. In particular, in pure GR, not only the general coordinate transformation, but also the rotations of local Lorentz frames are gauge symmetries. We also need to check whether there are ghosts or tachyons before we claim that the YM theory of the gauge symmetry of Sec.2 can be interpreted as a gravity theory. This will be the main issue to focus on below.

Nevertheless, motivated by this potential identification, we denote the covariant derivative as

$$
D_{a}=\hat{e}_{a}^{\mu} \partial_{\mu}
$$

where we used the notation

$$
\hat{e}_{a}{ }^{\mu} \equiv \delta_{a}^{\mu}+A_{a}{ }^{\mu} .
$$

The kinetic term of a scalar field is

$$
\eta^{a b} D_{a} \phi D_{b} \phi=\hat{g}^{\mu \nu} \partial_{\mu} \phi \partial_{\nu} \phi,
$$


where the effective metric $\hat{g}_{\mu \nu}$ naturally arises. It is defined by

$$
\hat{g}_{\mu \nu}=\hat{e}_{\mu}^{a} \eta_{a b} \hat{e}_{\nu}^{b}
$$

where $\hat{e}_{\mu}{ }^{a}$ is by definition the inverse of $\hat{e}_{a}{ }^{\mu}$.

\subsection{Field Strength vs Torsion}

The field strength of the non-Abelian gauge symmetry constructed above is

$$
F_{a b}(x) \equiv\left[D_{a}, D_{b}\right]=\partial_{a} A_{b}(x)-\partial_{b} A_{a}(x)+\left[A_{a}(x), A_{b}(x)\right] .
$$

In the representation $(23)$, it is

$$
F_{a b}(x)=F_{a b}^{(\lambda)}(x) \partial_{\lambda},
$$

where

$$
F_{a b}^{(\lambda)}(x)=\hat{e}_{a}^{\mu} \partial_{\mu} \hat{e}_{b}^{\lambda}-\hat{e}_{b}^{\mu} \partial_{\mu} \hat{e}_{a}^{\lambda} .
$$

With the analogy between $\hat{e}_{\mu}{ }^{a}$ and the vielbein $e_{\mu}{ }^{a}$, we define

$$
\hat{T}_{\mu \nu}^{\lambda} \equiv \hat{\Gamma}_{\mu \nu}^{\lambda}-\hat{\Gamma}_{\nu \mu}^{\lambda} .
$$

It is the torsion for the Weitzenböck connection

$$
\hat{\Gamma}_{\mu \nu}^{\lambda} \equiv \hat{e}_{a}^{\lambda} \partial_{\mu} \hat{e}_{\nu}^{a}
$$

used in teleparallel gravity when $\hat{e}_{\mu}{ }^{a}$ is identified with the vielbein $e_{\mu}{ }^{a}$. The field strength and the "torsion" are essentially the same quantity:

$$
F_{a b}^{(\lambda)}(x)=-\hat{e}_{a}^{\mu} \hat{e}_{b}^{\nu} \hat{T}_{\mu \nu}^{\lambda}
$$

if we think of $\hat{e}_{\mu}{ }^{a}$ and $\hat{e}_{a}{ }^{\mu}$ as the quantities used to switch between the two bases $\partial_{\mu}$ and $D_{a}$.

The "connection" (44) satisfies the relation

$$
D_{\mu} \hat{e}_{\nu}^{a} \equiv \partial_{\mu} \hat{e}_{\nu}^{a}-\hat{\Gamma}_{\mu \nu}^{\lambda} \hat{e}_{\lambda}^{a}=0
$$

and has zero "curvature":

$$
\hat{R} \equiv d \hat{\Gamma}-\Gamma \wedge \Gamma=0
$$

\subsection{Field-Dependent Killing Form}

An interesting feature of the algebra (16) for space-time diffeomorphism is that the Killing form (invariant inner product) has to be field-dependent.

For two elements of the Lie algebra $T_{f} \equiv f^{\mu}(x) \partial_{\mu}$ and $T_{f^{\prime}} \equiv f^{\prime \nu}(x) \partial_{\nu}$, it is clear that the Killing form should be

$$
\left\langle T_{f} \mid T_{f^{\prime}}\right\rangle=\int d^{D} x \sqrt{\hat{g}} f^{\mu}(x) \hat{g}_{\mu \nu}(x) f^{\prime \nu}(x)
$$


up to an overall normalization constant factor. Here the measure $\sqrt{\hat{g}}=\operatorname{det} \hat{e}_{\mu}{ }^{a}$ must be present to ensure that the integration is diffeomorphism-invariant.

The Killing form can be slightly simplified by a change of basis. Let us use the fielddependent basis $\left\{D_{a}\right\}$. The Killing form for two generators $T_{f}=f^{a} D_{a}$ and $T_{f^{\prime}}=f^{\prime b} D_{b}$ is

$$
\left\langle T_{f} \mid T_{f^{\prime}}\right\rangle=\int d^{D} x \operatorname{det} \hat{e} f^{a}(x) \eta_{a b}(x) f^{\prime b}(x),
$$

where $f^{a} \equiv f^{\mu} \hat{e}_{\mu}{ }^{a}$ and similarly for $f^{\prime b}$. (The factor of $\hat{g}_{\mu \nu}$ in (48) is replaced by $\eta_{a b}$.) In this basis, the structure constants are field-dependent:

$$
\left[D_{a}, D_{b}\right]=F_{a b}{ }^{c} D_{c}
$$

and the Jacobi identity (the consistency of the Lie algebra) is equivalent to the Bianchi identity of the field strength.

\section{$4 \quad \mathrm{YM}$ as Gravity}

\subsection{YM Action}

The YM action is given as the norm of the field strength:

$$
S_{Y M} \equiv \frac{1}{4 \kappa^{2}}\left\langle F_{a b} \mid F^{a b}\right\rangle=\int d^{D} x \frac{\operatorname{det} \hat{e}}{4 \kappa^{2}} F^{a b c} F_{a b c} .
$$

It is invariant under space-time diffeomorphism. However, it is not invariant under rotations of the local Lorentz frame:

$$
\hat{e}_{\mu}^{a}(x) \rightarrow \hat{e}_{\mu}^{\prime a}(x)=\omega^{a}{ }_{b}(x) \hat{e}_{\mu}^{b}(x)
$$

In the absence of the gauge symmetry of local Lorentz frame rotations, the variable $\hat{e}_{\mu}{ }^{a}$ contains more degrees of freedom than the genuine vielbein $e_{\mu}{ }^{a}$. (This is why we have used a hat to distinguish it from the vielbein.) The YM theory cannot be identified with pure GR.

To achieve a YM-like theory equivalent to Einstein's theory, we should utilize the fact that the internal space index $a$ (for the basis $D_{a}$ ) can be contracted with the coordinate index $a$. It allows us to introduce quadratic terms in addition to (51) in the action. The most general quadratic action is the superposition of three terms:

$$
S_{Y M-l i k e}=\int d^{D} x \frac{\operatorname{det} \hat{e}}{\kappa^{2}}\left[\frac{\lambda}{4} F^{a b c} F_{a b c}+\frac{\alpha}{4} F^{a b c} F_{a c b}-\frac{\beta}{2} F_{b}^{a b} F_{a c}{ }^{c}\right] .
$$

The action remains the same if we simultaneously scale $\kappa^{2}, \lambda, \alpha, \beta$ by the same factor. Up to this ambiguity, there is a unique choice of the parameters such that this action is invariant under local Lorentz rotations. 


\subsection{Teleparallel Gravity}

The action (53) is of a form resembling that of the teleparallel gravity, which is equivalent to Einstein's theory [10].

The teleparallel gravity has the interpretation as the gauge theory of translational symmetry. The gauge potential is essentially the vielbein:

$$
\mathcal{A}_{\mu}{ }^{a} \equiv e_{\mu}{ }^{a}-\delta_{\mu}{ }^{a}
$$

where $\delta_{\mu}{ }^{a}$ can be replaced by an arbitrary constant matrix. The torsion $T^{\lambda}{ }_{\mu \nu}$ of the Weitzenböck connection is essentially the Abelian field strength

$$
T_{\mu \nu}^{a}=\partial_{\mu} e_{\nu}^{a}-\partial_{\nu} e_{\mu}^{a}=\partial_{\mu} \mathcal{A}_{\nu}{ }^{a}-\partial_{\nu} \mathcal{A}_{\mu}{ }^{a} .
$$

Despite the fact that the field strength (42) of the generalized gauge symmetry of diffeomorphism is related to this field strength (55) by a mere change of basis in the tangent space, the gauge symmetries are totally different. The gauge symmetry is Abelian in the traditional interpretation of teleparallel gravity, while the diffeomorphism is of course non-Abelian.

The action of teleparallel gravity is

$$
S_{T P}=\int d^{D} x \frac{e}{2 \kappa^{2}}\left[\frac{1}{4} T^{\lambda \mu \nu} T_{\lambda \mu \nu}+\frac{1}{2} T^{\mu \nu \lambda} T_{\lambda \nu \mu}-T_{\lambda}^{\lambda \mu} T_{\mu \nu}^{\nu}\right],
$$

where indices are raised or lowered using the metric $g_{\mu \nu}=e_{\mu}{ }^{a} e_{\nu}{ }^{b} \eta_{a b}$ and $e=\sqrt{g}$ stands for the determinant of $e_{\mu}^{a}$. The Lagrangian of this action equals the Hilbert-Einstein Lagrangian up to a total derivative. That is,

$$
\mathcal{L}_{T P}=\frac{e}{2 \kappa^{2}} R^{(L C)}+\text { total derivatives, }
$$

where $R^{(L C)}$ is the scalar curvature for the (torsion-free) Levi-Civita connection. Even though the choices of connections are different, the teleparallel gravity action and the HilbertEinstein action give exactly the same field equation for the metric and so they are physically equivalent.

It is interesting that the inverse of $(I+\mathcal{A})$ (see (54)) for the potential $\mathcal{A}$ of the Abelian group of translations can be identified with $(I+A)$ for the gauge potential $A_{a}{ }^{\mu}$ of the non-Abelian gauge symmetry of general coordinate transformations.

The teleparallel gravity action (56) is equivalent to the action (53) for the choice of parameters $\lambda=1 / 2, \alpha=1, \beta=1$. It is

$$
S_{T P}=\int d^{D} x \frac{\operatorname{det} \hat{e}}{2 \kappa^{2}}\left[\frac{1}{4} F^{a b c} F_{a b c}+\frac{1}{2} F^{a b c} F_{c b a}-F^{a b}{ }_{b} F_{a c}{ }^{c}\right] .
$$

The first term is the YM action (51). The rest of the terms provide the unique combination so that the action is invariant under rotations of local Lorentz frames (52). The field $\hat{e}_{\mu}{ }^{a}$ can now be identified with the vielbein $e_{\mu}{ }^{a}$ in gravity, and the modified YM theory is equivalent to GR. 


\subsection{Metric, $B$-field and Dilaton}

While the action (58) is equivalent to pure GR, we investigate the most general quadratic action (53), which can be equivalently put in the form

$$
S=\int d^{D} x \frac{\operatorname{det} \hat{e}}{2 \kappa^{2}}\left[\frac{1}{2} F^{a b c} F_{a b c}-\frac{\alpha}{12}\left(F^{a b c}+F^{b c a}+F^{c a b}\right)\left(F_{a b c}+F_{b c a}+F_{c a b}\right)-\beta F_{b}^{a b} F_{a c}{ }^{c}\right],
$$

assuming that the coefficient of the YM term is non-zero. It is invariant under general coordinate transformations for arbitrary constants $\alpha, \beta$. (Compared with (53), $\lambda=1-\alpha / 2$.) The case of teleparallel gravity (56) corresponds to the choice $\alpha=\beta=1$.

For generic values of $\alpha, \beta$, local rotations of Lorentz frames are no longer gauge symmetries. With fewer gauge symmetries, there are more physical degrees of freedom in the theory. In $D$-dimensional space-time, the fundamental field $\hat{e}_{a}{ }^{\mu}$ has $D^{2}$ components. When the rotation of local Lorentz frames is a gauge symmetry, local Lorentz transformations identify $D(D-1) / 2$ components of $\hat{e}_{a}{ }^{\mu}$ as gauge artifacts, with the remaining $D(D+1) / 2$ components of $\hat{e}_{a}{ }^{\mu}$ to be matched with the $D(D+1) / 2$ independent components of the metric.

Tuning the values of $\alpha, \beta$ slightly away from 1 , we have $D(D-1) / 2$ of the components that can no longer be gauged away. The theory with generic values of $\alpha, \beta$ is expected to contain more physical fields in addition to the metric. For coefficients $\alpha, \beta$ with values not too different from 1, the theory is expected to be a gravity theory including matter fields. After all, the gauge symmetry of general coordinate transformation is always present.

In Einstein's theory of gravity, for a fluctuation of the metric

$$
g_{\mu \nu}=\eta_{\mu \nu}+h_{\mu \nu}+\cdots,
$$

one can choose the vielbein to be symmetric

$$
e_{\mu a}=\eta_{\mu a}+h_{\mu a} / 2+\cdots
$$

as a condition for the local Lorentz frame. Note that in the perturbation theory we are forced to mix the Latin and Greek indices as the space-time is Minkowskian at the lowest order. We will no longer distinguish the indices in the perturbative theory, and use both sets of labells $a, b, c, \cdots$ and $\mu, \nu, \cdots$ at will.

We decompose the field $A_{\mu a}$ into the symmetric part and the anti-symmetric part

$$
A_{a b}=\left(h_{a b}+B_{a b}\right) / 2
$$

where $h_{a b}$ is symmetric and $B_{a b}$ is anti-symmetric. We identify $h_{a b}$ as the fluctuation of the metric, and only the traceless part of $h_{a b}$ propagates in Einstein's theory. When the rotation of the local Lorentz frame is not a gauge symmetry, the trace part of $h_{a b}$ and the tensor $B_{a b}$ cannot be gauged away.

For the theory to be physically sensible, one has to check that there are no ghosts or tachyons. A necessary condition for linearized field equations of a rank-2 tensor field to 
be free of ghosts and tachyonic modes is that the anti-symmetric part of the tensor field is decoupled from the symmetric part [17]. The implication of this criterion to the general quadratic action (59) can be easily derived as follows. First, the cyclic combination

$$
F_{a b c}+F_{b c a}+F_{c a b}=\left(\partial_{a} B_{b c}+\partial_{b} B_{c a}+\partial_{c} B_{a b}\right)+\mathcal{O}\left(A^{2}\right)
$$

in the second term of the action (59) involves only the anti-symmetric tensor field $B_{a b}$ at the linearized level. (This was why we chose the peculiar form of the second term in the action (59).) Hence its coefficient $\alpha$ has no effect on the coupling between $h_{a b}$ and $B_{a b}$.

The first and third terms in the action (59) involve both $h_{a b}$ and $B_{a b}$, and the relative magnitude of their coefficients should be fixed to decouple $h_{a b}$ from $B_{a b}$. Since we know that pure GR is free of ghosts and tachyons, the ratio of these coefficients should be identical to that of the teleparallel gravity action (56). Consequently, the parameter $\beta$ should be fixed as [18]

$$
\beta=1
$$

It is still necessary to check that the kinetic terms are positive-definite. The parameter $\alpha$ is constrained by [18]

$$
\alpha<1
$$

for the kinetic term of the anti-symmetric field $B_{a b}$ to be positive-definite. These two conditions (64) and (65) ensures that the theory is ghost-free and tachyon-free at the free field level.

For this class of theories, the propagating modes of the traceless part of $h_{a b}$ should be interpreted as the graviton, and the trace part $h^{a}{ }_{a}$ as the dilation. There is also a rank-2 anti-symmetric field $B_{a b}$ whose gauge transformation at the lowest order is

$$
\delta B_{a b}=\partial_{a} \Lambda_{b}-\partial_{b} \Lambda_{a}+\mathcal{O}(A)
$$

One can define the covariant field strength of $B_{a b}$ as $\left(F_{a b c}+F_{b c a}+F_{c a b}\right)(63)$.

The dilaton can be viewed as a 0 -form gauge potential. In the Lorentz gauge

$$
\partial_{\mu} \hat{e}_{a}^{\mu}=0
$$

we have

$$
\begin{aligned}
F_{b}^{a b} & =\hat{e}^{a c} \partial_{c} \hat{e}_{b}^{b}-\hat{e}^{b c} \partial_{c} \hat{e}^{a}{ }_{b} \\
& =\frac{D}{2} \partial^{a} \hat{\phi}+\mathcal{O}\left(A^{2}\right),
\end{aligned}
$$

where

$$
\hat{\phi} \equiv h_{b}^{b} / D
$$

so that $F_{a b}{ }^{b}$ can be interpreted as the field strength of the dilaton. 


\subsection{Comparison with Other Formulations of Gravity}

At first sight, the formulation of gravity outlined above may appear reminiscent to other known formulations of gravity as a gauge theory. We have already commented above how the traditional interpretation of teleparallel gravity (as an Abelian gauge theory) is different from our formulation. There are also non-Abelian gauge theory formulations of gravity, e.g. Chern-Simons gravity in 3D [6], MacDowell-Mansouri gravity in 4D [7] and higher dimensional generalizations [9]. In these theories, the gauge potential is of the form

$$
A_{\mu}=e_{\mu}^{a} P_{a}+\omega_{\mu}^{a b} J_{a b}
$$

where $P_{a}$ and $J_{a b}$ are generators of the local Poincare algebra iso $(D-1,1)$ or the conformal algebra $s o(D-1,2)$. The components of the gauge potential are the vielbein $e_{\mu}{ }^{a}$ and the spin connection $\omega_{\mu}^{a b}$. In contract, the gauge potential in our theory is (in a certain representation)

$$
A_{a}=\left(\hat{e}_{a}^{\mu}-\delta_{a}^{\mu}\right) \partial_{\mu},
$$

where $\hat{e}_{a}^{\mu}$ might be identified with the inverse of the vielbein if the action respects the local Lorentz symmetry.

Formally, if we identify the translation generator $P_{a}$ in (70) with the derivative $\partial_{\mu}$ in (71), the gauge potential (70) resembles (71). However, more precisely, there are many important differences.

1. The generators $P_{a}$ (and $J_{a b}$ ) in (70) commute with functions on the base space, while the derivative in (71) does not.

2. The coefficient of $P_{a}$ is the vielbein, and that of $\partial_{\mu}$ is the inverse vielbein in the special case of teleparallel gravity. In general, $\hat{e}_{\mu}{ }^{a}$ includes more degrees of freedom than the inverse vielbein.

3. Eq. (70) is the usual potential associated with the "gauging" of a finite dimensional Lie group, while (71) is not the potential for gauging any global symmetry.

4. The field strength for (70) is given by the Riemann tensor, and that for (71) by the torsion of the Weitzenböck connection. A priori they are not related in any simple way as the Weitzenböck connection is not invariant under local Lorentz transformations while the Riemann tensor is.

5. Despite the fact that the Lagrangians for gravity are quadratic in the field strengths for both potentials (70) and (71). The former gives the Hilbert-Einstein action. Even in the special case of telelparallel gravity, the latter differs by a total derivative.

Supergravity theories are constructed [12] based on the YM-like theory for the gauge potential (70). It will be interesting to consider the supersymmetrization of the gauge symmetry of diffeomorphism and to derive the supergravity theory as an alternative formulation of supergravity. We leave this project for future publications. 


\section{Scattering Amplitudes}

In recent years, there has been amazing progress in the techniques of calculating scattering amplitudes, as well the understanding of their structures. Among them, a very interesting and mysterious structure is the connection between YM theory and gravity through the socalled double-copy procedure, which utilizes the color-kinematics duality (also known as the BCJ duality) [13]. According to Ref. [13], certain gravity theories are double copies of YM theories: the scattering amplitudes of gravity theories can be obtained from those of YM theories with color factors replaced by kinematic factors. In many cases this connection can find its origin in the open-closed string duality (the KLT duality [19]), although there are also other cases in which the string-theory origin is absent at this moment.

A complete off-shell field-theoretic explanation of this connection between gravity and YM theories, which applies only to on-shell amplitudes, may not be possible. But it is desirable to understand how much of the on-shell miracle can be understood in an off-shell theory. Earlier efforts in this direction include Refs. [20,21]. We propose that the formulation of gravity as a YM theory we constructed above may shed some new light on this problem.

\subsection{Heuristic Explanation}

Let us first re-examine the double-copy procedure to illustrate our idea. As the simplest example, for the pure YM theory, the color-ordered 3-point amplitude at tree-level is

$$
f_{a b c} n_{\lambda \mu \nu}^{(3)}(p, q,-(p+q))
$$

where $f_{a b c}$ is the structure constant and $n_{\lambda \mu \nu}^{(3)}(p, q,-(p+q))$ the kinematic factor

$$
n_{\lambda \mu \nu}^{(3)}(p, q, r) \equiv(p-q)_{\nu} \eta_{\lambda \mu}+(q-r)_{\lambda} \eta_{\mu \nu}+(r-p)_{\mu} \eta_{\nu \lambda}
$$

Here $(p, q, r)$ are the momenta of the 3 external legs, and $\lambda, \mu, \nu$ are Lorentz indices labelling the polarizations of the vector fields. The origin of this factor (73) is the 3 -point vertex

$$
\left[A_{\mu}, A_{\nu}\right]_{c} F_{(0)}^{\mu \nu c}=f_{a b c} A_{\mu}^{a} A_{\nu}^{b}\left(\partial^{\mu} A^{\nu c}-\partial^{\nu} A^{\mu c}\right)
$$

in the YM Lagrangian, with cyclic permutations of the three factors of $A$ contracted with three external legs, assuming that the basis of the Lie algebra is chosen such that $f_{a b c}=$ $f_{b c a}=f_{c a b}$. Here $F_{(0)}^{\mu \nu c} \equiv \partial^{\mu} A^{\nu c}-\partial^{\nu} A^{\mu c}$ is the field strength at the lowest order.

The double-copy procedure states that the replacement of $f_{a b c}$ by $n_{\lambda \mu \nu}^{(3)}(p, q,-(p+q))$ gives the 3 -point amplitude of the corresponding gravity theory. In other words, if there is a Lie algebra with indices $a=(\lambda, p)$ and structure constants $f_{a b c}$ given by $n_{\lambda \mu \nu}^{(3)}(p, q,-(p+q))$, the YM theory would agree with GR at least for 3-point amplitudes. Yet one can check that this choice of structure constants does not satisfy the Jacobi identity.

The color-kinematics duality and the double-copy procedure applies to all higher-point amplitudes. For 4-point amplitudes at the tree level, the kinematic factor of color-ordered 
amplitudes is ${ }^{2}$, skematically,

$$
n_{i}^{(4)}=n_{i}^{(3)} n_{i}^{(3)}+m_{i}^{(4)} \quad(i=s, t, u)
$$

where the first term on the right hand side is the contribution from 3-point vertices, and the second term from the 4-point interaction in the YM theory. (The index values $s, t, u$ are labels for the $s, t$ and $u$ channels of the Feynman diagrams for tree-level 4-point scattering amplitudes.) The kinematic factors $n_{i}^{(4)}$ satisfy a linear relation

$$
n_{s}^{(4)}+n_{t}^{(4)}+n_{u}^{(4)}=0
$$

analogous to the Jacobi identity for the color factor (which is quadratic in the structure constants). The relation above would be equivalent to the Jacobi identify for $n^{(3)}$ interpreted as structure constants if the terms $m_{i}^{(4)}$ were absent. The presence of $m_{i}^{(4)}$ is the evidence that $n^{(3)}$ cannot be used as structure constants.

Often the relation between YM and GR indicated by the double-copy procedure is symbolically represented as $(\mathrm{YM})^{2}=(\mathrm{GR})$. This expression is actually misleading, because neither the color factors or the propagators are squared in the gravity theory. Instead, the identification of color factors with kinematic factors (e.g. $f_{a b c}$ with $n_{\lambda \mu \nu}(p, q,-(p+q))$ for tree-level 3-point amplitudes) identifies YM directly with GR. It is more appropriate to use (YM)' $=\mathrm{GR}$ as the symbolic representation of this connection. The prime on (YM) indicates the modification of YM theory by the replacement of color factors by kinematic factors.

Since the color factors are composed of structure constants of the gauge group, we are naturally led to consider the possibility of gauge symmetries with structure constants involving kinematic factors. This was precisely what we did in Sec.2, which led to new formulations of gravity theories as generalized YM theories in Sec.3

Apparently, the hope for a direct matching between structure constants and the kinematic factors is too naive. First, as the 3-point amplitude is defined on-shell, the structure constant can be different from $n_{\lambda \mu \nu}(p, q,-(p+q))$ when it is off-shell. Secondly, even if the 3-point amplitudes agree with structure constant, it is not clear if higher-point amplitudes will automatically agree with the corresponding color factors, as there will be different on-shell conditions at work. (In fact, eq.(77) says that the structure constants for 4-point amplitudes are not to be given by the structure constants for 3-point amplitudes due to the extra term $m_{i}^{(4)}$.) In general, as the BCJ duality only holds on-shell, the correspondence between

${ }^{2}$ More explicitly [22],

$$
n_{s}^{(4)}=\epsilon_{1}^{\lambda} \epsilon_{2}^{\mu} \epsilon_{3}^{\nu} \epsilon_{4}^{\sigma} n_{\sigma \nu \rho}^{(3)}\left(-p_{4},-p_{3}, p_{3}+p_{4}\right) n_{\lambda \mu}^{(3) \rho}\left(p_{1}, p_{2},-p_{1}-p_{2}\right)+m_{s}^{(4)},
$$

where $p_{i}^{\mu}, \epsilon_{i}^{\mu}(i=1,2,3,4)$ are the momenta and polarization vectors of the external legs, $s=\left(p_{1}+p_{2}\right)^{2}$ and

$$
m_{s}^{(4)} \equiv s\left[\left(\epsilon_{1} \cdot \epsilon_{4}\right)\left(\epsilon_{2} \cdot \epsilon_{3}\right)-\left(\epsilon_{1} \cdot \epsilon_{3}\right)\left(\epsilon_{2} \cdot \epsilon_{4}\right)\right] .
$$

(The choice of $m_{s}^{(4)}$ is not unique.) The other two kinematic factors $n_{t}^{(4)}, n_{u}^{(4)}$ can be obtained by permutations of external legs. 
structure constants and kinematic factors in $n$-point amplitudes is different for different $n$, and it is unclear if there exists an off-shell generalization. It is also highly nontrivial how such on-shell correspondences can be implemented efficiently in a field-theoretic approach. Hence we leave the search for a field-theoretic proof of the validity of the double-copy procedure for future works. Nevertheless, the color-kinematics duality and double-copy procedure motivate us to explore YM theories with Lie algebras involving kinematic factors, and it does lead to a connection between YM theories and gravity theories at the level of Lagrangians as we have shown in Sec.3.

It will be interesting to see whether the calculation of scattering amplitudes is simplified in this YM-like formulation of gravity, compared with the calculation based on the HilbertEinstein action. It will be even more interesting to see if we are getting closer to the simplified on-shell results obtained via the BCJ duality.

To gain some intuition about how far or close our theory is to the concise results of the BCJ duality, let us comment on the skematic structure of the 3-point scattering amplitude (72). The kinematic factor $n$ comes from the factor $F_{(0)}$, as the only object involving derivatives in the cubic term $f A A F_{(0)}$ (74) of the YM action. This suggests that, to replace the color factor $f$ by $n$, we should have $f \sim F_{(0)}$ to the lowest order in $A$. But this is precisely what we have: the structure constant (50) in the basis of $D_{a}$ is the field strength $F$ !

In the following, we will compute more carefully the 3-point vertices of the YM-like formulation of gravity, and see that the calculation is much simpler than the calculation based on the Hilbert-Einstein action (which involves around 100 terms). We leave higherpoint scattering amplitudes for the future.

Incidentally, although the Chern-Simons theory in 3D [6] and the MacDowell-Mansouri theory in 4D [7] are also YM-type formulations of gravity, they are first order formulations of GR. One has to first solve the connection in terms of the vielbein before calculating any scattering amplitudes of gravitons. The calculation in those theories is not simpler than a direct computation from the Hilbert-Einstein action.

\subsection{Perturbative Expansion in $A$}

In this section, we focus on the 3-point vertices relevant for the 3-graviton scattering. We consider 3-point vertices of the action (59) for the traceless part of $h_{\mu \nu}$.

First, using (63), one can easily see that the second term in the action (59) is

$$
\frac{\alpha}{12}\left(F^{a b c}+F^{b c a}+F^{c a b}\right)\left(F_{a b c}+F_{b c a}+F_{c a b}\right)=\frac{\alpha}{3} H^{(0) a b c} H_{a b c}^{(0)}+\mathcal{O}\left(H^{(0)} A^{2}\right)+\mathcal{O}\left(A^{4}\right),(79
$$

where $H_{a b c}^{(0)}$ is the field strength of the anti-symmetric tensor field $B_{a b}$ defined at the lowest order:

$$
H_{a b c}^{(0)} \equiv \partial_{a} B_{b c}+\partial_{b} B_{c a}+\partial_{c} B_{a b} .
$$

A vertex operator involving only external legs of $h$ appears at $\mathcal{O}\left(A^{4}\right)$ or higher. The 3-point vertices of $h_{\mu \nu}$ is thus independent of the parameter $\alpha$. 
Secondly, the third term in (59) is the square of

$$
F_{a b}^{b}=\partial_{a} A_{b}{ }^{b}-\partial_{b} A_{a}{ }^{b}+\mathcal{O}\left(A^{2}\right)
$$

where the first term involves the trace of $h_{\mu \nu}$, and the second term vanishes if we impose the gauge-fixing condition

$$
\partial^{b} h_{a b}=0
$$

for the graviton field. As a result, in this gauge (82), the third term of the action (59) is also irrelevant to the 3-point vertex for the traceless part of $h_{\mu \nu}$.

Furthermore, the overall measure of integration is

$$
\operatorname{det} e=1+h_{a}{ }^{a}+\mathcal{O}\left(A^{2}\right)
$$

which is also irrelevant for our consideration.

The 3-point vertices for the graviton can thus only come from the YM Lagrangian, and there are only two terms

$$
\mathcal{L}^{(3)} \equiv F_{(0)}^{a b c}\left(\left[A_{a}, A_{b}\right]_{c}-F_{a b}^{(0)} d A_{d c}\right)
$$

where

$$
\left[A_{a}, A_{b}\right]_{c} \equiv A_{a}{ }^{d} \partial_{d} A_{b c}-A_{b}{ }^{d} \partial_{d} A_{a c}
$$

and $F_{a b c}^{(0)}$ is the field strength at the zero-th order

$$
F_{a b c}^{(0)} \equiv \partial_{a} A_{b c}-\partial_{b} A_{a c}
$$

The first term of (84) comes from the Lie algebra structure of this generalized YM theory. The second term arises due to the field-dependent inner-product of the Lie algebra.

Near the end of Sec.5.1, we discussed how the formulation of gravity as a generalized YM theory can heuristically explain the double-copy procedure for 3-point amplitudes at tree level. Eq.(84) is the exact expression of the heuristic expression $f A A F_{(0)} \sim F_{(0)} A F_{(0)}$ there. It may seem that there is a small discrepancy between $F_{(0)}\left([A, A]+A F_{(0)}\right)(84)$ and $F_{(0)} A F_{(0)}$. But recall that the structure constant $f_{a b c}$ is assumed to be cyclic in the doublecopy procedure (and in our heuristic discussion in Sec.5.1), while $F_{(0)}^{a b c}$ is not. The exact expression (84) is in fact of the form of $F_{(0)} A F_{(0)}$ but additional terms that have some of the indices permuted. In Sec.5.3 below, we will see a simpler and more direct match with the discussions in Sec.5.1.

The 3-point vertices for the gravitons are therefore

$$
\left(\partial^{a} h^{b c}-\partial^{b} h^{a c}\right)\left[h_{a d} \partial^{d} h_{b c}-h_{b d} \partial^{d} h_{a c}-\left(\partial_{a} h_{b}{ }^{d}-\partial_{b} h_{a}{ }^{d}\right) h_{d c}\right]
$$

This is already a very simple expression, especially if we compare it with the expression obtained from the Hilbert-Einstein action. But the expression can be even further simplified as we will shown below. 


\subsection{Perturbative Expansion in $\hat{A}$}

It is more economic, at least at the lowest order, to use the variable $\hat{A}_{\mu a}$ defined by

$$
\hat{e}_{\mu}^{a}=\delta_{\mu}^{a}+\hat{A}_{\mu}^{a}
$$

where $\hat{e}_{\mu}{ }^{a}$ is the inverse of $\hat{e}_{a}{ }^{\mu}(37)$. The new variable $\hat{A}_{\mu}{ }^{a}$ is merely a field redefinition of $A_{a}{ }^{\mu}$. They are related via

$$
A_{a}{ }^{\mu}=\hat{e}_{a}{ }^{\mu}-\delta_{a}^{\mu}=-\hat{A}_{a}{ }^{\mu}+\hat{A}_{a}{ }^{\nu} \hat{A}_{\nu}^{\mu}+\mathcal{O}\left(A^{3}\right) .
$$

Therefore

$$
h_{a b} \simeq-\hat{h}_{a b}+\cdots .
$$

Up to sign, the physical (on-shell) amplitudes of $h_{a b}$ and $\hat{h}_{a b}$ should agree. As it is suggested by the notation, we have decomposed $\hat{A}$ as

$$
\hat{A}_{a b}=\hat{h}_{a b} / 2+\hat{B}_{a b}
$$

where $\hat{h}_{a b}$ is symmetric and $\hat{B}_{a b}$ is anti-symmetric. The trace part of $\hat{h}_{a b}$ is denoted ${ }^{3}$

$$
\hat{\phi} \equiv \frac{1}{D} \hat{h}_{a}^{a} .
$$

The effective metric (39) is

$$
\hat{g}_{\mu \nu}=\hat{e}_{\mu}^{a} \eta_{a b} \hat{e}_{\nu}^{b} \simeq \eta_{\mu \nu}+\hat{h}_{a b}+2 \eta_{a b} \hat{\phi}+\mathcal{O}\left(A^{2}\right)
$$

We also have

$$
\operatorname{tr} \hat{e} \equiv \hat{e}_{\mu}^{a} \delta_{a}^{\mu}=D\left(1+\hat{\phi}+\mathcal{O}\left(A^{2}\right)\right)
$$

and thus

$$
\operatorname{det} \hat{e}=1+D \hat{\phi}+\mathcal{O}\left(A^{2}\right)
$$

One can ignore the integration measure det $\hat{e}$ when the 3 -point vertex under consideration does not involve $\hat{\phi}$ as an external leg.

Expanding the field strength in powers of $\hat{A}$, we have

$$
F_{a b c}=-\hat{e}_{a}^{\mu} \hat{e}_{b}^{\nu}\left(\partial_{\mu} \hat{A}_{\nu c}-\partial_{\nu} \hat{A}_{\mu c}\right)
$$

Then,

$$
\frac{1}{2} F_{a b c} F^{a b c}=\frac{1}{2} \hat{F}_{a b c}^{(0)} \hat{F}^{(0) a b c}-2 \hat{F}^{(0) a b c} \hat{A}_{a}{ }^{d} \hat{F}_{d b c}^{(0)}+\mathcal{O}\left(A^{4}\right)
$$

where

$$
\hat{F}_{\mu \nu a}^{(0)} \equiv \partial_{\mu} \hat{A}_{\nu a}-\partial_{\nu} \hat{A}_{\mu a} .
$$

\footnotetext{
3 As we have learned from pure GR, the trace part of the fluctuation of the metric is not a physical propagating mode. Hence we should identify the trace part of $\hat{A}_{a b}$ (and $A_{a b}$ ) as an independent scalar field.
} 
Similar to the perturbative expansion in terms of $A$, the second and third terms in the action (59) do not contribute to 3-point interactions of the traceless part of $h_{a b}$.

It is remarkable that for this action (59) there is a single 3-point vertex for graviton interaction (the second term in (97))

$$
\hat{\mathcal{L}}^{(3)}=-2 \hat{F}^{(0) a b c} \hat{A}_{a}{ }^{d} \hat{F}_{d b c}^{(0)} .
$$

This is precisely of the form $F_{(0)} A F_{(0)}$ needed to explain the double-copy procedure at tree level as we discussed in Sec.5.1. This is of course also a significant simplification compared with the usual expression of GR.

\section{Higher-Form Gauge Symmetries}

In the above we have focused on the gauge symmetries with 1-form potentials and 0 -form gauge parameters. We can also apply the same notion of generalization to gauge symmetries with higher-form gauge potentials. The basic ideas of our generalization are the following:

1. The symmetry generators do not have to be factorizable in the form

$$
T(\{f, a\})=\sum_{n} f_{n}(x) T_{a_{n}}
$$

where $f_{n}(x)$ 's are functions on the base space and $T_{a}$ 's are elements of a finite-dimensional Lie algebra.

2. Even if the symmetry generators are formally factorable in the form (100), the objects $T_{a}$ 's do not have to commute with base-space functions.

If we take a given principal bundle as a classical manifold and deform its algebra of functions so that it becomes noncommutative, in general, this noncommutative space is not the tensor product of a group and a noncommutative base space. This is a way to construct examples of the comment 1 above.

As an example of the comment 2, even though the gauge symmetry constructed in Sec.2 has generators of the form (100) with $T_{a}=\partial_{a}$, these $T_{a}$ 's should not be interpreted as generators of a finite-dimensional Lie algebra (otherwise the Lie algebra is Abelian) and they do not commute with space-time functions.

For higher-form gauge symmetries, only the Abelian case is well understood. There is no consensus on the definition of non-Abelian higher-form gauge symmetries, ${ }^{4}$ and concrete examples are scarce. Due to this reason, our discussion below cannot be very precise. It is commonly speculated that there is something analogous to the Lie algebra whose elements replace $T_{a}$ in the factorized formula (100) of a gauge transformation generator. The analogue

\footnotetext{
4 The mathematical structure for the symmetry of a 2-form gauge potential is called a non-Abelian gerbe. But there are different versions of its definition.
} 
of our generalization of gauge symmetry for higher-form gauge symmetries is then referring to a violation of that factorization.

The Nambu-Poisson gauge theory $[23,24]$ is one of the few examples of non-Abelian higher-form gauge theories. It was used to describe an M5-brane in a large $C$-field background. It can be viewed as the covariant lift of the Poisson limit of the noncommutative gauge symmetry for a D4-brane in large $B$-field background to a higher dimension. The Nambu-Poisson gauge symmetry has a 2-form potential with 1-form transformation parameters. Its gauge group is the non-Abelian group of volume-preserving-diffeomorphisms. It can be viewed as an example of generalized gauge symmetry for higher form potentials.

Incidentally, the fact that the gauge algebra involves kinematic factors is also the reason why it is possible for higher-form gauge symmetries to be non-Abelian. Higher-form global symmetries are always Abelian [25]. Hence an ordinary procedure of "gauging" the global symmetry by introducing space-time dependence to the generators in a way analogous to eq.(100) can never result in a non-Abelian gauge symmetry (unless the space-time coordinates are noncommutative). Conversely, for a non-Abelian higher-form gauge symmetry, when the transformation parameters are restricted to be constant, all kinematic factors become trivial, and the symmetry algebra becomes Abelian. The Nambu-Poisson gauge symmetry is clearly an example of this fact. The noncommutative $U(1)$ gauge symmetry is the lower-form analogue.

Another example of non-Abelian gauge symmetry with a 2-form gauge potential is the low energy effective theory for multiple M5-branes proposed in Ref. [26,27]. The M5-branes are compactified on a circle, and the gauge transformation laws distinguish zero-modes from KK modes. The distinct treatment on zero-modes and KK modes can be viewed as a dependence on the kinematic factor (whether the momentum is zero or not), and so it is also an example of the generalized gauge theory for higher forms.

There are other examples of non-Abelian gauge symmetry with higher-form gauge potentials [28], in addition those mentioned above. It will be interesting to explore further how the idea promoted above on generalized gauge symmetry will help the construction of a mathematical framework for non-Abelian higher-form gauge theories.

\section{Acknowledgement}

The author would like to thank Chong-Sun Chu, Kazuo Hosomichi, Yu-Tin Huang, Takeo Inami for their interest and discussions. This work is supported in part by the National Science Council, Taiwan, R.O.C. and by the National Taiwan University. 


\section{References}

[1] P. M. Ho and S. Ramgoolam, "Higher dimensional geometries from matrix brane constructions," Nucl. Phys. B 627, 266 (2002) [hep-th/0111278].

[2] P. M. Ho and C. T. Ma, "S-Duality for D3-Brane in NS-NS and R-R Backgrounds," JHEP 1411, 142 (2014) [arXiv:1311.3393 [hep-th]].

[3] R. Utiyama, "Invariant theoretical interpretation of interaction," Phys. Rev. 101, 1597 (1956).

[4] T. W. B. Kibble, "Lorentz invariance and the gravitational field," J. Math. Phys. 2, 212 (1961).

[5] D. W. Sciama, "On the analogy between charge and spin in general relativity," in Recent Developments in General Relativity (Oxford: Pergamon) p.415 (1962).

[6] E. Witten, "(2+1)-Dimensional Gravity as an Exactly Soluble System," Nucl. Phys. B 311, 46 (1988).

[7] S. W. MacDowell and F. Mansouri, "Unified Geometric Theory of Gravity and Supergravity," Phys. Rev. Lett. 38, 739 (1977) [Erratum-ibid. 38, 1376 (1977)].

[8] K. S. Stelle and P. C. West, "Spontaneously Broken De Sitter Symmetry and the Gravitational Holonomy Group," Phys. Rev. D 21, 1466 (1980).

[9] M. A. Vasiliev, "Cubic interactions of bosonic higher spin gauge fields in AdS(5)," Nucl. Phys. B 616, 106 (2001) [Erratum-ibid. B 652, 407 (2003)] [hep-th/0106200].

[10] For an introduction to teleparallel gravity, see: V. C. De Andrade, L. C. T. Guillen and J. G. Pereira, "Teleparallel gravity: An Overview," gr-qc/0011087, or: R. Aldrovandi and J. G. Pereira, "Teleparallel Gravity : An Introduction," Springer (ISBN 978-94-0075143-9).

[11] I. Cortese and J. A. Garcia, "Emergent Noncommutative gravity from a consistent deformation of gauge theory," Phys. Rev. D 81, 105016 (2010) [arXiv:1001.4180 [hepth]].

[12] For a review, see: P. Van Nieuwenhuizen, "Supergravity," Phys. Rept. 68, 189 (1981).

[13] Z. Bern, J. J. M. Carrasco and H. Johansson, "New Relations for Gauge-Theory Amplitudes," Phys. Rev. D 78, 085011 (2008) [arXiv:0805.3993 [hep-ph]]. Z. Bern, 
J. J. M. Carrasco and H. Johansson, "Perturbative Quantum Gravity as a Double Copy of Gauge Theory," Phys. Rev. Lett. 105, 061602 (2010) [arXiv:1004.0476 [hep-th]]. Z. Bern, J. J. M. Carrasco, L. J. Dixon, H. Johansson and R. Roiban, "The Complete Four-Loop Four-Point Amplitude in N=4 Super-Yang-Mills Theory," Phys. Rev. D 82, 125040 (2010) [arXiv:1008.3327 [hep-th]]. Z. Bern, S. Davies, T. Dennen, Y. t. Huang and J. Nohle, "Color-Kinematics Duality for Pure Yang-Mills and Gravity at One and Two Loops," arXiv:1303.6605 [hep-th].

[14] Y. M. Cho, "Einstein Lagrangian as the Translational Yang-Mills Lagrangian," Phys. Rev. D 14, 2521 (1976).

[15] A. Connes, "Noncommutative geometry," ISBN-9780121858605.

[16] P. Salgado, G. Rubilar, J. Crisostomo and S. del Campo, "A note about teleparallel supergravity," Eur. Phys. J. C 44, 587 (2005).

[17] P. Van Nieuwenhuizen, "On ghost-free tensor lagrangians and linearized gravitation," Nucl. Phys. B 60, 478 (1973).

[18] F. Mueller-Hoissen and J. Nitsch, "Teleparallelism - A Viable Theory Of Gravity?," Phys. Rev. D 28, 718 (1983).

[19] H. Kawai, D. C. Lewellen and S. H. H. Tye, Nucl. Phys. B 269, 1 (1986).

[20] N. E. J. Bjerrum-Bohr, P. H. Damgaard, R. Monteiro and D. O'Connell, "Algebras for Amplitudes," JHEP 1206, 061 (2012) [arXiv:1203.0944 [hep-th]].

[21] R. Monteiro, D. O'Connell and C. D. White, arXiv:1410.0239 [hep-th].

[22] D. p. Zhu, "Zeros in Scattering Amplitudes and the Structure of Nonabelian Gauge Theories," Phys. Rev. D 22, 2266 (1980).

[23] P. M. Ho and Y. Matsuo, "M5 from M2," JHEP 0806, 105 (2008) [arXiv:0804.3629 [hep-th]]. P. M. Ho, Y. Imamura, Y. Matsuo and S. Shiba, "M5-brane in three-form flux and multiple M2-branes," JHEP 0808, 014 (2008) [arXiv:0805.2898 [hep-th]]. P. M. Ho, "A Concise Review on M5-brane in Large C-Field Background," Chin. J. Phys. 48, 1 (2010) [arXiv:0912.0445 [hep-th]].

[24] C. H. Chen, K. Furuuchi, P. M. Ho and T. Takimi, "More on the Nambu-Poisson M5-brane Theory: Scaling limit, background independence and an all order solution to the Seiberg-Witten map," JHEP 1010, 100 (2010) [arXiv:1006.5291 [hep-th]]. 
P. M. Ho and C. H. Yeh, "D-brane in R-R Field Background," JHEP 1103, 143 (2011) [arXiv:1101.4054 [hep-th]]. P. M. Ho and C. T. Ma, "Effective Action for Dp-Brane in Large RR (p-1)-Form Background," JHEP 1305, 056 (2013) [arXiv:1302.6919 [hep-th]]. P. M. Ho, "Gauge Symmetries from Nambu-Poisson Brackets," Universe 1, 46 (2013).

[25] D. Gaiotto, A. Kapustin, N. Seiberg and B. Willett, "Generalized Global Symmetries," arXiv:1412.5148 [hep-th].

[26] P. M. Ho, K. W. Huang and Y. Matsuo, "A Non-Abelian Self-Dual Gauge Theory in 5+1 Dimensions," JHEP 1107, 021 (2011) [arXiv:1104.4040 [hep-th]]. K. -W. Huang, "Non-Abelian Chiral 2-Form and M5-Branes," arXiv:1206.3983 [hep-th]. P. M. Ho and Y. Matsuo, "Aspects of Effective Theory for Multiple M5-Branes Compactified On Circle," arXiv:1409.4060 [hep-th].

[27] P. M. Ho and Y. Matsuo, "Note on non-Abelian two-form gauge fields," JHEP 1209, 075 (2012) [arXiv:1206.5643 [hep-th]].

[28] P. Aschieri and B. Jurco, "Gerbes, M5-brane anomalies and E(8) gauge theory," JHEP 0410, 068 (2004) [hep-th/0409200]. H. Samtleben, E. Sezgin and R. Wimmer, "(1,0) superconformal models in six dimensions," JHEP 1112, 062 (2011) [arXiv:1108.4060 [hep-th]]. S. Palmer and C. Saemann, "M-brane Models from Non-Abelian Gerbes," arXiv:1203.5757 [hep-th]. C. Saemann and M. Wolf, "Non-Abelian Tensor Multiplet Equations from Twistor Space," arXiv:1205.3108 [hep-th]. F. Bonetti, T. W. Grimm and S. Hohenegger, "Non-Abelian Tensor Towers and $(2,0)$ Superconformal Theories," arXiv:1209.3017 [hep-th]. C. Saemann and M. Wolf, "Six-Dimensional Superconformal Field Theories from Principal 3-Bundles over Twistor Space," arXiv:1305.4870 [hep-th]. S. Palmer and C. Sämann, "Six-Dimensional $(1,0)$ Superconformal Models and Higher Gauge Theory," J. Math. Phys. 54, 113509 (2013) [arXiv:1308.2622 [hep-th]]. S. Palmer, "Higher Gauge Theory and M-Theory," arXiv:1407.0298 [hep-th]. B. Jurco, C. Saemann and M. Wolf, "Semistrict Higher Gauge Theory," arXiv:1403.7185 [hep-th]. 